\title{
Implementation of information and analysis support of the industrial enterprise's logistical management based on the tools of the fuzzy set theory
}

\author{
G.M. Greyz ${ }^{1, *}$ \\ ${ }^{1}$ South Ural State University, Chelyabinsk, Russia
}

\begin{abstract}
Management of industrial enterprises' logistical systems is based on application of rather heterogeneous and not always certain information. Presence of different types of uncertainty in the complex hierarchical system of industrial enterprises' logistical management gives grounds for analysis support of management solutions based on the fuzzy set theory. Use of the fuzzy set theory allows to link together and adequately consider all the necessary heterogeneous information. In this regard, information on functioning of the logistical system must be presented in a specific form as membership functions. It is justified in the article that the tools of the fuzzy set theory can be applied for description of parameters of the industrial enterprises' logistical system and justification of decision-taking in the sphere of logistical management. Within the framework of the system of information and analysis support of industrial enterprises' logistical management it is proposed to use tools of problem "determination of the fuzzy set image" and its variety - "definition of the sub direct fuzzy set image" in order to choose the best variant of combination of key efficiency indicators of logistical management complying with the present complex of criteria. Application of the fuzzy set theory also allows to determine fuzzy values of factors, as a result of which the enterprise's logistical system has obtained the existing or objective set of features. For analysis of factors influencing the key efficiency indicators of the industrial enterprise's logistical management it is proposed to use tools of problem "definition of the fuzzy set pre-image at a fuzzy binary relation".
\end{abstract}

\section{Introduction}

Development and taking of management decisions in the logistical management system must be based on obtaining and processing of specific information messages. The author has proved that in order to increase efficiency of the industrial enterprise's logistical management the necessary information must be generated and processed by a special system of information and analysis support of logistical management [2].

According to the author, the system of information and analysis support of logistical management must ensure: - selection of a system of key parameters-characteristics of functioning of the industrial enterprise's logistical system; - agreement of strategic and tactical objectives of logistical management with the adopted system of indicators;

- conversion of the selected indicators into a form necessary for their processing and analysis by the system of information and analysis support of logistical management;

- analysis of actual values of parameters-characteristics of functioning of the industrial enterprise's logistical system for their compliance with the system of target criteria;

- identification of a potential connection of deviations of the logistical system's actual parameters and the management decisions taken;
- analytical check (forecasting) of consequences of the planned management decisions in the sphere of logistical management for their compliance with the preset system of target criteria.

According to the author, the system of information and analysis support of logistical management is a key link of interfunctional coordination in the industrial enterprise management system.

\section{Theoretical framework and literature review}

In order to ensure stable development of the logistical system it must have such system property as homeostasis [9]. The author believes that the system of information and analysis support of logistical management constitutes one of the conditions for achievement of the homeostasis state by the logistical system ensuring its adaptability to external and internal disturbances. Efficient functioning of the industrial enterprise's logistical system is maintained on account of entry and processing of relevant information in its management system.

Corresponding author: ggreyz09@mail.ru 
In the author's concept the system of information and analysis support of the industrial enterprise's logistical achievement of homeostasis by the enterprise's logistical system and meant for: measurement of key indicators of the industrial enterprise's logistical system; complex evaluation of logistical management efficiency based on the criteria entered in the system; monitoring of consequences of management decisions in the logistical sphere.

Information and analysis support of the industrial enterprise's logistical management is provided by this system on account of available inverse relationship with the logistical system of the enterprise itself and the logistical system of the next level including the industrial enterprise and its counterparties.

Subject to the author's methodology, the system of information and analysis support is a necessary link of the industrial enterprise's logistical management, which obtains the necessary information on the enterprise's logistics activity, converts it into a specific form necessary for analysis, analyses it in terms of effectiveness and uses the obtained results for evaluation of efficiency of current management decisions in the sphere of logistical management and monitoring of consequences of planned management decisions.

The system of information and analysis support of the industrial enterprise's logistical management consists of: subsystem of formation and evaluation of key indicators of logistical management, matrix model of information and analysis support of logistical management and monitoring of management decisions in the sphere of logistical management.

The author of this research believes that the subsystem of formation and evaluation of key indicators of logistical management must be based on the concept of balanced scorecard. The offered subsystem of formation and evaluation of key indicators of logistical management provides for a transfer from verbally stated objectives to relevant groups of indicators of logistical management and indicators themselves. A set of indicators establishes selection of a certain interpretation of objective statement from a potential range of these statements. The indicators, which reflect a specific objective, are united into relevant groups by objectives.

The analysis conducted by the author of this research has shown that currently there is no ready set of groups of key indicators of logistical management and relevant criteria suitable for application in the author's system of information and analysis support of logistical management.

In the course of the research it has been determined that in order to increase mobility of initial data collection and to simplify their analysis for entry in the subsystem of formation and evaluation of key indicators of the industrial enterprise's logistical management it is necessary to use six groups of key indicators. They, primarily, include such groups of indicators as financial indicators, as well as time, expenses, customer satisfaction and inventories.

It should be also noted that the methodological approach to creation of a complex of information and analysis support of the industrial enterprise's logistical management is defined as a system contributing to

flow process management offered in the research assumes application of the sixth group of key indicators, which contains the author's logistical indicators reflecting specificity of the industrial enterprise's activities. These indicators reflect the level of inefficiency of material flow organization at the considered industrial enterprise.

In several cases, including at carrying out activity in the sphere of logistics, there appears a need to take decisions in conditions of lacking complete and certain (clear) information. For some of such situations classical mathematics assumes application of the probability theory. However, fuzziness of some phenomena does not always have a probabilistic nature. Besides, it is often impossible to apply the probability theory due to lacking test data. Use of the probability theory as applied to uncertain quantities does not give a proper effect, whereas the source of uncertainty at decision-taking is not a contingency but fuzziness $[2,4,6,13]$. In order to solve this problem it was necessary to introduce a new definition of uncertainty, and one of such definitions was provided by the fuzzy set theory offered by Professor of the University of California Lotfi A. Zadeh [5,20]. His work "Fuzzy Sets" [20], which appeared in 1965, laid the basis of the fuzzy set theory and establishment of intellectual systems able to interact adequately with the human being.

As opposed to the customary set theory, where there are only two potential memberships of the subset element: it can be either a member or not a member of this subset, in the fuzzy set theory fuzziness is formalized by introduction of the definition of the degree of membership of an element in a fuzzy set.

The fuzzy set theory was further developed in the 1980 -s, when computer systems using fuzzy control algorithms started to appear. The fuzzy set theory laid the basis for building complex process control systems, as well as found application in home appliances, diagnostic and other expert systems [7,10,14,15,17-19].

The fuzzy set theory allows to convert expert estimates in terms of the natural language into the quantitative form, which allows to simulate economic entities based on opinions of experts observing these or similar entities.

Sufficient heterogeneous information is needed for logistical system management. These are values of different parameters, their potential variation parameters, as well as verbal information obtained from experts in the form of so-called linguistic variables.

Presence of different types of uncertainty in the complex hierarchical system of industrial enterprises' logistical management gives grounds for information and analysis support of management decisions in these systems based on the fuzzy set theory, which allows to take justified decisions in view of different types of uncertainty $[1,8,11,16]$.

Application of the fuzzy set theory allows to link together and adequately consider all the necessary heterogeneous information. At the same time, all the information on functioning of the logistical system and 
objective functions must be presented in a specific form as membership functions.

The circumstance, that at description of different systems, including logistical systems, effective values of their functioning parameters are mostly fuzzy - there is no actual border between one effective value and other values close to it, counts in favor of application of the fuzzy set theory. An insignificant change of the system parameter usually leads to a small change in efficiency, therefore, application of the fuzzy subset membership function better corresponds to a real situation than application of preset (clear) parameters of logistical system efficiency.

In several cases at complex system management there is no need to take a clear decision for each point of time, whereas expenses on obtaining of information necessary for this decision and change of system parameters can exceed the effect obtained from this management decision. There frequently appears a situation, when not clear values but the most probable estimate of these values, as well as their potential change range can be set for several system parameters. Conditions and specificity of management problems generally permit fuzzy decisions. A significant factor of setting fuzzy conditions and objectives is complexity of multi-level logistical systems of industrial enterprises and presence of numerous links between their subsystems. Setting of clear restrictions and objectives is actually possible only for simple single-level systems.

In view of the above-mentioned, tools of the fuzzy set theory can be applied for description of parameters of the industrial enterprises' logistical system and lay the basis of the industrial enterprise's logistical model and justification of decision-taking in the sphere of logistical management. At creation of this model constituting the nucleus of the author's system of information and analysis support of the industrial enterprise's logistical management [2], the author has used the concept that any enterprise being an open logistical system is in the process of continuous changing. The enterprise's internal processes and their interaction (or, at least, interinfluence) and changing environmental conditions precondition continuous changes in various parameters of the enterprise's activity, including logistical parameters. Measurement of these parameters reflects their current values at a certain point of their changes and is a unique photo recording "floating", not exactly clear values of the dynamic process. The author believes that this uncertainty justifies application of the fuzzy set theory for study and evaluation of logistical systems.

\section{Materials and methods}

\section{A. Methods Description}

Parameters of the logistical system, which values are fuzzy sets, are used for reflection of the economic reality and simulation of the economic entity. Within the framework of the industrial enterprise's logistical model tools of the fuzzy set theory are used for solution of two tasks:
1) Analysis and evaluation of key indicators of the industrial enterprise's logistical management efficiency

2) Evaluation and monitoring of management decisions in the logistical sphere

Let us review, what opportunities are provided by the fuzzy set theory for solution of the set problems. At analysis and description of elements of the fuzzy set theory necessary for conducting of this research the material presented in [12] was taken as a basis.

In several cases for evaluation of efficiency of any activity, including logistical management, the set of criteria and calculation of the integrating efficiency indicator are used. However, calculation of the integrating efficiency indicator does not always solve the problem on evaluation of the logistical system management optimality degree. In several cases calculation of the integrating indicator does not give an answer to the question on optimality of this or that combination of key parameters of the logistical system. One and the same value of the complex logistical management efficiency indicator can be obtained at different combinations of parameters of the developed evaluation system. At the same time, specific features of an individual industrial enterprise result in specificity of combinations of objective evaluation criteria. The problem lies is selection of one best combination complying with the preset complex of criteria out of several combinations of the logistical system parameters with close integral values. For such selection within the framework of the methodology of information and analysis support of the industrial enterprise's logistical management the author proposes to use the tools of problem "determination of the fuzzy set image at fuzzy binary relation" and its variety - "determination of the subdirect fuzzy set image".

Statement of problem "determination of the fuzzy set image" can be presented as follows: there are several economic entities, each of which has a set of specific properties (features), and there is a criterial set of these features, in accordance with which the economic entities are evaluated. After solution of this problem we can find an economic entity, which has at least one feature from the criterial set.

Within the framework of this field of research sets of indicators formed by the subsystem of formation of key indicators of the industrial enterprise's logistical management are used as economic entities [3]. Such sets consist of a set reflecting the current state of the logistical system, indicators of past and future (forecast) periods. The criterial set of features is based on the requirements to be met by the key indicators of logistical management converted into fuzzy sets (ratings). After solution of this problem we can evaluate, which of the considered sets of ratings has a larger degree of membership in the criterial set of requirements.

Let us consider this problem in terms of the fuzzy set theory. It is necessary to find an image of fuzzy set $A$ (criterial set of features $x$ ) in set $Y$ (economic entities $y$ ), provided that we know fuzzy binary relation $R$ from set $X$ to set $Y$ (sets of properties (features) of economic entities). After solution of this problem we find 
membership degree $q$ for each element $y$ in the image of fuzzy set $A$ at the preset fuzzy binary relation (presentation) from set $X$ to set $Y-R$. Values of features $x$, binary relations $r$ and membership degree $q$ of element $y$ in the image of fuzzy set $A$ subject to the laws of the fuzzy set theory must be within interval $[0 ; 1]$, i.e., the closer value $q$ to one, the larger degree of compliance of the complex of the economic entity's properties with the preset criterial set of features $A$.

In general terms, fuzzy set $B$ constituting a direct image of set $A$ can be written as $B=A \downarrow R$. - selection symbol $(\max -\min )$. In the matrix form this problem can be solved as follows:

$$
\left(p_{1}, p_{2}, \ldots, p_{n}\right) \bullet\left[\begin{array}{cccc}
r_{11} & r_{12} & \ldots & r_{1 m} \\
r_{21} & r_{22} & \ldots & r_{2 m} \\
\ldots & \ldots & \ldots & \ldots \\
r_{n 1} & r_{n 2} & \ldots & r_{n m}
\end{array}\right]=\left(q_{1}, q_{2}, \ldots, q_{n}\right)
$$

where $p_{1}, p_{2}, \ldots, p_{n}$ - degree of membership of features $x_{1}, x_{2}, \ldots, x_{n}$ in the fuzzy set (criterial set), which is written as $A=\left\{\left(x_{1} \mid p_{1}\right) ;\left(x_{2} \mid p_{2}\right) ; \ldots\left(x_{n} \mid p_{n}\right)\right\}$;

$r_{11} \ldots r_{n m}$ - elements of matrix $R$ (fuzzy binary relation from set $X$ to set $Y$ ), which preset fuzzy values of features $x$ for elements $y$ (economic entities) of fuzzy set $Y$;

$q_{1}, q_{2}, \ldots, q_{m}-$ degrees of membership of elements $y_{1}, y_{2}, \ldots, y_{m}$ in the image of fuzzy set $A$, which can be interpreted as a degree of conformity of the complex of economical entities' properties with the preset criterial set of values of these features;

$n$ - number of features $x$;

$m$ - number of elements $y$ (economic entities);

Solutions of expression (1) in the coordinate form are

$$
\begin{aligned}
& \max \left(\min \left(p_{1} ; r_{11}\right) ; \ldots \min \left(p_{n} ; r_{n 1}\right)\right)=q_{1} \\
& \max \left(\min \left(p_{1} ; r_{1 m}\right) ; \ldots \min \left(p_{n} ; r_{n m}\right)\right)=q_{m}
\end{aligned}
$$

Based on the calculation results by formulas (2) we obtain set $B=A \backslash R=\left\{\left(y_{1} \mid q_{1}\right) ;\left(y_{2} \mid q_{2}\right) ; \ldots ;\left(y_{m} \mid q_{m}\right)\right\}$ on the ground of which we can evaluate, which economic entity $y$ has a larger degree of membership in the image of fuzzy set $A$. The larger value $q$ (potential range of values $[0 ; 1]$ ), the larger the compliance of the economic entity with the preset criterial set of features $A$ by availability and value of properties.

Apart from problem "determination of the fuzzy set image", for analysis and evaluation of key efficiency indicators of the industrial enterprise's logistical management we can use a variety of this problem - "determination of the subdirect fuzzy set image". The difference is that as a result of solution of this problem we determine a set of indicators, which has not one but all the features specified in the criterial set and which best of all corresponds to this set by the complex of its properties.
In general terms, fuzzy set $B$ constituting a subdirect image of set $A$ can be written as $B=A<R$. In view of the introduced symbols in the matrix form this problem can be solved as follows:

$$
\left(p_{1}, p_{2}, \ldots, p_{n}\right)<\left[\begin{array}{cccc}
r_{11} & r_{12} & \ldots & r_{1 m} \\
r_{21} & r_{22} & \ldots & r_{2 m} \\
\ldots & \ldots & \ldots & \ldots \\
r_{n 1} & r_{n 2} & \ldots & r_{n m}
\end{array}\right]=\left(q_{1}, q_{2}, \ldots, q_{n}\right)
$$

Values of symbols of expression (3) coincide with the values presented in expression (1). The difference lies in introduction of operation 4 , which content becomes clear from recording of expression (3) in the coordinate form

$$
\begin{gathered}
\min \left(\left(p_{1}-r_{11}\right) ; \ldots ;\left(p_{n} \square r_{n 1}\right)\right)=q_{1} \\
\ldots \ldots \ldots \ldots \ldots \ldots \ldots \ldots \ldots \ldots \ldots \ldots \ldots \\
\min \left(\left(p_{1}-r_{1 m}\right) ; \ldots ;\left(p_{n}-r_{n m}\right)\right)=q_{m}
\end{gathered}
$$

The so-called cut-off operation $(\boldsymbol{\square})$ can be presented as follows:

$$
p \mathbf{\square} r= \begin{cases}1 & \text { at } p \leq r \\ r & \text { at } p>r\end{cases}
$$

Based on the calculation results by formulas (4) we obtain set $B=A<R=\left\{\left(y_{1} \mid q_{1}\right) ;\left(y_{2} \mid q_{2}\right) ; \ldots\left(y_{m} \mid q_{m}\right)\right\}$, on the ground of which we can evaluate, which economic entity $y$ has a larger degree of membership in the subdirect image of fuzzy set $A$. Analogously to determination of the image, the larger value $q$, the larger the compliance of the economic entity with the preset criterial set of features $A$ by the complex of properties. Within the framework of the matrix model we can also analyze factors influencing the key efficiency indicators of the industrial enterprise's logistical management. For this purpose, within the framework of the methodology of information and analysis support of the industrial enterprise's logistical management the author proposes to use tools of problem "determination of the fuzzy set pre-image at a fuzzy binary relation".

Statement of problem "determination of the fuzzy set pre-image" can be presented as follows: there is a set of factors, which values are unknown, and an economic entity having a set of specific properties (features), and there are known cause-and-effect relations between the factors of influence on the economic entity and the properties (features) of this entity. Upon solution of this problem we have to restore fuzzy values of factors, as a result of which influence the economic entity has obtained the existing or desirable (target) set of features.

Within the framework of the system of information and analysis support of logistical management problem "determination of the fuzzy set pre-image" is transformed as follows: a) we select a set of factors significant for logistical management (fuzzy set $A$ ), b) we set in a fuzzy form the degree of influence of the selected factors on the key efficiency indicators of logistical management (cause-and-effect relations between the factors of influence on the economic entity and the properties (features) of this entity), i.e. in this case relations of transfer from fuzzy set $A$ to fuzzy set $B$, (fuzzy binary relation $R$ of transfer from set $X$ to set $Y$ ), c) we determine current and preset target values of key 
efficiency indicators of logistical management in the form of ratings by groups of indicators (set of specific properties (features) y) (known fuzzy set B). Solution of this problem results in fuzzy values of factors, under the influence of which the logistical management system has obtained the existing or desirable (target) set of indicators.

Let us consider this problem in terms of the fuzzy set theory. It is necessary to restore fuzzy set $A$ on the basis of known fuzzy set $B$, if we know fuzzy binary relation $R$ of transfer from set $X$ to set $Y$, i.e. in this case relations of transfer of fuzzy set $A$ to fuzzy set $B$. There can exist the following relations between fuzzy sets $B$ and $A: B=$ $A \downarrow R$ (determination of -pre-image), $B=A \backslash R$ (determination of 4 -pre-image) and $B=A>R$ (determination of -pre-image) [6]. Selection of this or that model determines the relevant function of membership of fuzzy set $A$ and its calculation procedure.

Let us consider calculation of $\boldsymbol{1}$-pre-image. In the matrix form this problem can be solved as follows:

$$
\left(q_{1}, q_{2}, \ldots, q_{m}\right) \triangleright\left[\begin{array}{cccc}
r_{11} & r_{12} & \ldots & r_{1 m} \\
r_{21} & r_{22} & \ldots & r_{2 m} \\
\ldots & \ldots & \ldots & \ldots \\
r_{n 1} & r_{n 2} & \ldots & r_{n m}
\end{array}\right]=\left(p_{1}^{\bullet}, p_{2}^{\bullet}, \ldots, p_{n}^{\bullet}\right)
$$

where $p_{1}^{\bullet}, p_{2}^{\bullet}, \ldots, p_{n}^{\bullet}$ - fuzzy values of factors, as a result of which influence the logistical management system has obtained or could have obtained key efficiency parameters $q_{1}, q_{2}, \ldots, q_{m}$;

$r_{11} \ldots r_{n m}$ - elements of matrix $R$ in the fuzzy form preset the degree of influence of the selected factors $p$ of the key efficiency indicators of logistical management $q$.

$q_{1}, q_{2}, \ldots, q_{m}-$ fuzzy values of key efficiency parameters of the logistical management system.

degrees of membership of elements $y_{1}, y_{2}, \ldots, y_{m}$ in the image of fuzzy set $A$, which can be interpreted as a degree of conformity of the complex of the economic entities' features with the preset criterial set of values of these features;

$n$ - number of factors $x$;

$m$ - number of elements $y$ (key efficiency parameters);

Let us write the content of operation from expression (6) in the coordinate form on the analogy of expression (4)

$$
\begin{aligned}
& \min \left(\left(r_{11}-q_{1}\right) ;\left(r_{12}-q_{2}\right) ; \ldots\left(r_{1 m}-q_{m}\right)\right)=p_{1}^{\dagger}
\end{aligned}
$$

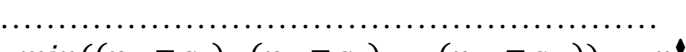

$$
\begin{aligned}
& \min \left(\left(r_{n 1}-q_{1}\right) ;\left(r_{n 2}-q_{2}\right) ; \ldots\left(r_{n m}-q_{m}\right)\right)=p_{n}^{\prime}
\end{aligned}
$$

The cut-off operation ( $\boldsymbol{\nabla})$, on the analogy with expression (5) can be presented as follows:

$$
r \backsim q= \begin{cases}1 & \text { при } r \leq q \\ q & \text { при } r>q\end{cases}
$$

Based on the calculation results by formulas (7) and (8) we restore the initial set $(\bullet$ - pre-image)

$A=B-R=\left\{\left(x_{1} \mid p_{1}^{\dagger}\right) ;\left(x_{2} \mid p_{2}^{\dagger}\right) ; \ldots\left(x_{n} \mid p_{n}^{\dagger}\right)\right\} \quad$ which after application of fuzzy relation $R$ was transferred to fuzzy set $B=\left\{\left(y_{1} \mid q_{1}\right) ;\left(y_{2} \mid q_{2}\right) ; \ldots\left(y_{m} \mid q_{m}\right)\right\}$.
Let us consider implementation of the second function of the matrix model - evaluation and monitoring of management decisions in the logistical sphere.

For implementation of this function within the framework of information and analysis support of the industrial enterprise's logistical management it is proposed to use the tools of problem "fuzzy inference models". According to the author, these tools of the fuzzy set theory can be efficiently used for monitoring of management decisions in the logistical sphere.

The content of this transfer can be presented as follows. The inference basis is formed by statement $r=$ 《if $a$, then $b \gg$, which is called implication in classical logics. In the fuzzy set theory statement $r$ is transformed into 《if $A$, then $B$ », where $A$ and $B$ are fuzzy sets interconnected by fuzzy relation $R$. This fuzzy relation is also called implication and denoted by symbol $A \Rightarrow B$.

After determination of the specific function of implication $A \Rightarrow B$ it becomes possible depending on input fuzzy set $A^{+}$to build output fuzzy set (effect) $B^{+}=A^{+} *(A \Rightarrow B)$.

In accordance with the author's approach the algorithm of the fuzzy inference model application is adapted to the sphere of information and analysis support of logistical management. Logical premise $\mathrm{A}+$ is a specific set of management actions, each of which has a different degree of "power" (intensity) preset by the fuzzy values within range $[0,1]$.

According to the author, such management actions $\left(X_{i}\right)$ in the sphere of logistical management include:

- operating-calendar planning of finished product output;

- operating process control;

- maintaining of quality standards;

- inventory control and management at all production stages;

- implementation of logistical information systems;

- investments in production automation;

- implementation of advanced cost management systems ("ABC-costing" or "target costing" or "kaizen costing"); - investments in R\&D in the sphere of logistics, etc.

In this case the complex of results of these management decisions can be presented as effect $B^{+}$.

The following output parameters of the industrial enterprise's logistical system are considered to be results of these management actions (efforts) $\left(Y_{i}\right)$ :

- improvement of product quality;

- cutting of the production lead time;

- performance improvement;

- cutting of logistical costs;

- inventory minimization (optimization) at all production stages;

- cost reduction;

- profit mark-up, etc.

Logistical concept or opinion of a group of experts must be used as implication $A \Rightarrow B$ in the form of fuzzy relation $R$. This approach allows to make forecasts based on the opinion of several groups of experts, and aggregation operation must be used to obtain an overall conclusion. 
Let us consider in detail the procedure of finding effect $B^{+}$of the complex of management actions $A^{+}$. In order to simplify description of the algorithm let us restrict complex $A^{+}$by five potential management actions, and their result $B^{+}$- also by five output parameters.

Then, the output management decisions will take the form of fuzzy set $A^{+}=\left\{\left(x_{1} \mid p_{1}\right) ;\left(x_{2} \mid p_{2}\right)\left(x_{3} \mid p_{3}\right)\left(x_{4} \mid p_{4}\right)\left(x_{5} \mid p_{5}\right)\right\}$. Let us assume that for finding of implication $A \Rightarrow B$ in the form of fuzzy relation $R$ the opinion of two experts (group of experts) is used. Their fuzzy sets have the following form $<<$ if $A_{i}$, then $B_{i}>>, \quad i=1,2$ and can be presented as follows

$A_{1}=\left\{\left(x_{1} \mid a_{1}^{1}\right) ;\left(x_{2} \mid a_{2}^{1}\right) ;\left(x_{3} \mid a_{3}^{1}\right) ;\left(x_{4} \mid a_{4}^{1}\right) ;\left(x_{5} \mid a_{5}^{1}\right)\right\}$. $B_{1}=\left\{\left(y_{1} \mid b_{1}^{1}\right) ;\left(y_{2} \mid b_{2}^{1}\right) ;\left(y_{3} \mid b_{3}^{1}\right) ;\left(y_{4} \mid b_{4}^{1}\right) ;\left(y_{5} \mid b_{5}^{1}\right)\right\}$.

$A_{2}=\left\{\left(x_{1} \mid a_{1}^{2}\right) ;\left(x_{2} \mid a_{2}^{2}\right) ;\left(x_{3} \mid a_{3}^{2}\right) ;\left(x_{4} \mid a_{4}^{2}\right) ;\left(x_{5} \mid a_{5}^{2}\right)\right\}$. $B_{2}=\left\{\left(y_{1} \mid b_{1}^{2}\right) ;\left(y_{2} \mid b_{2}^{2}\right) ;\left(y_{3} \mid b_{3}^{2}\right) ;\left(y_{4} \mid b_{4}^{2}\right) ;\left(y_{5} \mid b_{5}^{2}\right)\right\}$.

For building of the implication we use function $\theta(a, b)=a \mathbf{\square} b$ and present implication $A_{i} \Rightarrow$ $B_{i}=A_{i} \square B_{i}$ in the form of matrixes

$$
\begin{aligned}
A_{1} \square B_{1} & =\left(\begin{array}{ccc}
r_{11}^{1} & \cdots & r_{15}^{1} \\
\vdots & \ddots & \vdots \\
r_{51}^{1} & \cdots & r_{55}^{1}
\end{array}\right) \\
A_{2} \square B_{2} & =\left(\begin{array}{ccc}
r_{11}^{2} & \cdots & r_{15}^{2} \\
\vdots & \ddots & \vdots \\
r_{51}^{2} & \cdots & r_{55}^{2}
\end{array}\right)
\end{aligned}
$$

Matrix elements are as follows:

$r_{11}^{1}=a_{1}^{1}-b_{1}^{1} ; r_{12}^{1}=a_{1}^{1} \backsim b_{2}^{1} ; \ldots ; r_{55}^{1}=a_{5}^{1} \square b_{5}^{1}$ for matrix (10) and analogously for matrix (11). The rule of the cutoff operation $(\boldsymbol{\square})$, for this variant of comparison can be presented as follows:

$$
a \mathbf{\square} b= \begin{cases}1 & \text { at } a \leq \mathrm{b} \\ b & \text { at } a>b\end{cases}
$$

Then, on the basis of matrixes (10), (11) and output management decision $A^{+}$we will build local inferences $B_{1}^{+}$and $B_{2}^{+}$. As an inference rule let us take $*=\boldsymbol{\bullet}$.

$$
\begin{gathered}
\left(p_{1} ; p_{2} ; \ldots ; p_{5)} \diamond\left(\begin{array}{ccc}
r_{11}^{1} & \cdots & r_{15}^{1} \\
\vdots & \ddots & \vdots \\
r_{51}^{1} & \cdots & r_{55}^{1}
\end{array}\right)=\left(q_{1}^{1} ; q_{2}^{1} ; \ldots ; q_{5}^{1}\right)\right. \\
\left(p_{1} ; p_{2} ; \ldots ; p_{5)} \diamond\left(\begin{array}{ccc}
r_{11}^{2} & \cdots & r_{15}^{2} \\
\vdots & \ddots & \vdots \\
r_{51}^{2} & \cdots & r_{55}^{2}
\end{array}\right)=\left(q_{1}^{2} ; q_{2}^{2} ; \ldots ; q_{5}^{2}\right)\right.
\end{gathered}
$$

The solutions of the expression (13) in the coordinate form are the following expressions:

$$
\begin{aligned}
& \max \left(\min \left(p_{1} ; r_{11}^{1}\right) ; \ldots \min \left(p_{5} ; r_{51}^{1}\right)\right)=q_{1}^{1} \\
& \max \left(\min \left(p_{1} ; r_{15}^{1}\right) ; \ldots \min \left(p_{5} ; r_{55}^{1}\right)\right)=q_{5}^{1}
\end{aligned}
$$

Values $q_{1}^{2} \ldots q_{5}^{2}$ are determined analogously. Then, we will obtain fuzzy sets, which determine the result of the action of the management decision taken

$$
\begin{aligned}
& B_{(1)}^{+}=\left\{\left(y_{1} \mid q_{1}^{\text {min }}\right) ;\left(y_{2} \mid q_{2}^{\text {min }}\right) ; \ldots\left(y_{5} \mid q_{5}^{\text {min }}\right)\right\} \\
& B_{(2)}^{+}=\left\{\left(y_{1} \mid q_{1}^{\text {max }}\right) ;\left(y_{2} \mid q_{2}^{\text {max }}\right) ; \ldots\left(y_{5} \mid q_{5}^{\text {max }}\right)\right\}
\end{aligned}
$$

Values $q^{\text {min }}$ and $q^{\text {max }}$ are selected by comparison of resulting sets $q$ from formulas (13) and (14) for determination of local inferences.

Fuzzy inference models allow not only to determine the membership function for effect $B$ of fuzzy expression $A \Rightarrow B$, but also to solve a reverse problem: by setting fuzzy set $B$ as objective we can find input set $A$, as a complex of management actions in the logistical sphere. In this case the problem is realized as finding of the pre-image of fuzzy set $B$ (prime cause of obtaining the base set of key indicators).

\section{Conclusions}

Thus, it has been justified that within the system of information and analytical support of logistics management of an industrial enterprise the inventory of the fuzzy sets theory can be used for reaching the following tasks:

1. Multicriterial assessment of effectiveness of logistics management according to the key parameters of the logistics system and selection of the best combination of these parameters, that corresponds to the determined set of criteria.

2. On the basis of the determined causal relationships between the impacts of the logistics system and properties (evidences) of this system: reconstruction of fuzzy values of factors whose impacts stipulate the present or desired (aimed) set of properties of the logistics system.

3. On the basis of the accepted logistics concept or the opinion of an expert group: analytical prediction of the results received due to the impact of a certain set of managerial actions on the logistics system.

The work was supported by Act 211 Government of the Russian Federation, contract № 02.A03.21.0011

\section{References}

1. A.E. Altunin, M.V. Semukhin, Models and Algorithms of Decision Taking in Fuzzy Conditions: monograph (Publishing house of Tyumen State University, Tyumen, 2002)

2. G.M. Greyz, Bulletin of the South Ural State University, Series: Economics and Management, 8, 3, 121-127 (2014)

3. G.M. Greyz, V.M. Katochkov, V.V. Volozhanin, Bulletin of the South Ural State University. Series: Economics and Management, 8, 3, 128135 (2014) 
4. L.A. Gusev, I.M. Smirnova, Automatics and Telemechanics, 5, 66-85 (1973)

5. L.A. Zadeh, Definition of Linguistic Variable and its Application to Making of Approximate Solutions (Mir, Moscow, 1976)

6. L.A. Zadeh, Fuzzy Sets and their Application in Image Recognition and Cluster Analysis, Classification and Cluster: collection (Mir, Moscow, 1980)

7. A. Kaufman, Introduction to the Theory of Fuzzy Sets (Radio and Communication, Moscow, 1982)

8. B.L Kuchin, A.E. Altunin, Gas Supply System Management in Complicated Operating Conditions (Nedra, Moscow, 1987)

9. L.B. Mirotin, I.E. Tashbaev, System Analysis in Logistics (Ekzamen, Moscow, 2002)

10. S.A. Orlovskiy, Problems of Decision Taking at Fuzzy Initial Information (Nauka, Moscow, 1980)
11. M.V. Semukhin, Fuzzy Set Theory. Study guide (Tyumen State University, Tyumen, 1999)

12. V.I. Ukhobotov, Selected Chapters of the Fuzzy Set Theory: manual (Publishing house of Chelyabinsk State University, Chelyabinsk, 2011)

13. R.E. Bellman, M. Gierts, Inform. Sci., 5, 2, 149156 (1973)

14. S.S.L. Chang, Kybernetes, 6, 203-208 (1977)

15. D. Dubois, Prade Fuzzy Sets and Systems: Theory and Applications (Acad. Press, New York, 1980)

16. W.Y. Kickert, Automatica, 12, 4, 301-308 (1976)

17. H. Tanaka, K. Asai, IEEE Trans. Syst. Maan and Cybern, 2, 325-328 (1984)

18. B. Urban, V. Hansel, Proc. Fuzzy inf., Marseille, 313-320 (1983)

19. R.R. Yager, J. of Cybern, 10, 1-18 (1980)

20. L.A. Zadeh, Information and Control, 8, 3, 338353 (1965) 